\title{
Optimal titration is important to maximize the beneficial effects of vagal nerve stimulation on chronic heart failure
}

\author{
Akiko Nishizaki, MD
}

Department of Cardiovascular Medicine, Kyushu University Graduate School of Medical Sciences,

3-1-1 Maidashi, Higashi-ku, Fukuoka 812-8582, Japan.

nishizak@cardiol.med.kyushu-u.ac.jp

Kazuo Sakamoto, MD, PhD

Department of Cardiovascular Medicine, Saiseikai General Hospital,

1-3-46 Tenjin, Chu-ou-ku, Fukuoka 810-0001, Japan.

kazuos@cardiol.med.kyushu-u.ac.jp

Keita Saku, MD, PhD

Department of Therapeutic Regulation of Cardiovascular Homeostasis, Center for Disruptive Cardiovascular Medicine, Kyushu University,

3-1-1 Maidashi, Higashi-ku, Fukuoka 812-8582, Japan.

saku@ cardiol.med.kyushu-u.ac.jp

Kazuya Hosokawa, MD, PhD

Department of Cardiovascular Medicine, Kyushu University Graduate School of Medical Sciences,

3-1-1 Maidashi, Higashi-ku, Fukuoka 812-8582, Japan.

ncvchoso@cardiol.med.kyushu-u.ac.jp

Takafumi Sakamoto, MD, PhD 
Title

Department of Cardiovascular Medicine, Kyushu University Graduate School of Medical Sciences,

3-1-1 Maidashi, Higashi-ku, Fukuoka 812-8582, Japan.

tsaka@cardiol.med.kyushu-u.ac.jp

Yasuhiro Oga, MD

Department of Cardiovascular Medicine, Kyushu University Graduate School of Medical Sciences

3-1-1 Maidashi, Higashi-ku, Fukuoka 812-8582, Japan.

ogayasu@ cardiol.med.kyushu-u.ac.jp

Takuya Akashi

Department of Therapeutic Regulation of Cardiovascular Homeostasis, Center for Disruptive Cardiovascular Medicine, Kyushu University,

3-1-1 Maidashi, Higashi-ku, Fukuoka 812-8582, Japan.

akashi-t@cardiol.med.kyushu-u.ac.jp

Yoshinori Murayama

Department of Cardiovascular Medicine, Kyushu University Graduate School of Medical Sciences,

3-1-1 Maidashi, Higashi-ku, Fukuoka 812-8582, Japan.

murayama@cardiol.med.kyushu-u.ac.jp

Takuya Kishi, MD, PhD

Department of Therapeutic Regulation of Cardiovascular Homeostasis, Center for Disruptive Cardiovascular Medicine, Kyushu University,

3-1-1 Maidashi, Higashi-ku, Fukuoka 812-8582, Japan.

tkishi@cardiol.med.kyushu-u.ac.jp 
Title

Tomomi Ide, MD, PhD

Department of Cardiovascular Medicine, Kyushu University Graduate School of Medical Sciences,

3-1-1 Maidashi, Higashi-ku, Fukuoka 812-8582, Japan.

tomomi_I@cardiol.med.kyushu-u.ac.jp

Kenji Sunagawa, MD, PhD

Department of Therapeutic Regulation of Cardiovascular Homeostasis, Center for Disruptive Cardiovascular Medicine, Kyushu University,

3-1-1 Maidashi, Higashi-ku, Fukuoka 812-8582, Japan.

sunagawa@ cardiol.med.kyushu-u.ac.jp

Running head: Dose response of vagal nerve stimulation in chronic heart failure

\section{Corresponding author:}

Akiko Nishizaki

Department of Cardiovascular Medicine, Kyushu University Graduate School of Medical Sciences,

3-1-1 Maidashi, Higashi-ku, Fukuoka 812-8582, Japan.

Tel: $+81-92-642-5360$

Fax: +81-92-642-5374

E-mail: nishizak@ cardiol.med.kyushu-u.ac.jp 
Abstract

\section{$1 \quad$ Abstract}

\section{Background}

3 Although vagal nerve stimulation (VNS) benefits patients with chronic heart failure

4 (CHF), the optimal dose of VNS remains unknown. In clinical trials, adverse symptoms

5 limited up-titration. In this study, we evaluated the impact of various voltages of VNS

6 which were titrated below symptom threshold on cardiac function and CHF parameters

7 in rat myocardial infarction (MI) models.

$8 \quad$ Methods and Results

9 We randomly allocated MI rats to vagal (VNS, $n=41)$ and sham (Sham, $n=16$ )

10 stimulation groups. We stimulated the right vagal nerve with $20 \mathrm{~Hz}$ at three different

11 voltages for 4 weeks. We defined Max as the highest voltage that did not evoke any 12 symptom, Half as 1/2 of Max and Quarter as 1/4 of Max. All three groups significantly

13 reduced biventricular weight compared to Sham $(p<0.05)$. In contrast, only Half

14 decreased left ventricular (LV) end-diastolic pressure (Half: 17.5 \pm 2.0 , Sham: $24.2 \pm 1.2$

$15 \mathrm{mmHg}, p<0.05$ ), and increased LV ejection fraction (Half: $37.9 \pm 3.1$, Sham: $28.4 \pm 2.3 \%$, $16 p<0.05$ ) and LV maximum $+\mathrm{dP} / \mathrm{dt}$ (Half: 5918.6 \pm 2.0 , Sham: $5001.2 \pm 563.2 \mathrm{mmHg} / \mathrm{s}$, $17 p<0.05$ ). The number of large vagal nerve fibers was reduced with Max (Max: 18 163.1 \pm 43.0 , Sham: $360.0 \pm 61.6$ counts/bundle, $p<0.05$ ), indicating the significant neural 19 damage by VNS.

20 Conclusion

21 The optimal titration of VNS would maximize benefits for CHF and minimize adverse 22 effects.

24 Keywords: Vagal Nerve Stimulation, Chronic Heart Failure, Myocardial Infarction 


\section{Introduction}

27 Chronic heart failure (CHF) has a poor prognosis and is one of major public health concerns. Although new therapeutic strategies for CHF have been proposed in the last

29 decades, approximately one-half of CHF patients die within 5 years ${ }^{1}$. Since the 30 imbalance between sympathetic and parasympathetic nervous systems plays a major 31 role in the pathogenesis of $\mathrm{CHF}^{2}$, there is increasing clinical interest in 32 neuro-modulation therapy that alters the autonomic imbalance.

Since Li et al. ${ }^{3}$ reported that vagal nerve stimulation (VNS) markedly improved the survival of CHF rats, many animal studies had shown the favorable effects of VNS on CHF, such as decreases in heart rate and oxygen consumption ${ }^{4}$, reduction in

36 inflammation through activation of nicotinic receptors ${ }^{5}$, attenuation of norepinephrine 37 spillover in the left ventricle $(\mathrm{LV})^{6}$, suppression of free radical generation ${ }^{7}$ and sympatho-inhibition by activating the afferent $\operatorname{arm}^{8}$. Subsequent to these animal studies, a few clinical trials of VNS therapy for CHF were conducted but the outcomes were 40 inconsistent among trials ${ }^{9-11}$. Thus the efficacy of VNS for CHF in humans remains controversial. A non-randomized clinical trial exploring VNS in 32 NYHA classes II-IV CHF patients found that VNS improved quality of life, exercise capacity and LV

43 ejection fraction ${ }^{9}$. In contrast, the first randomized sham-controlled trial that investigated right-sided VNS for CHF patients (NECTAR-HF) failed to demonstrate significant beneficial effects on cardiac remodeling or functional capacity ${ }^{10}$. The results of NECTAR-HF raised many questions such as patient selection, synergistic effects of medications and the setting of electrical stimulation. Especially, incomplete understanding of the appropriate dosing of VNS makes it difficult to maximize the beneficial effects, while minimizing the adverse effects. 
Introduction

In animals studies, the dose of VNS were chosen at levels that reduce heart

51 rate $^{3}$. In contrast, the clinical trials showed that VNS-induced symptoms limited

52 up-titration of the stimulating dose; consequently heart rate was not reduced in most

53 cases $^{9,10}$. On the other hand, a recent investigation in dogs revealed that VNS improved

$54 \mathrm{CHF}$ independent of heart rate reduction ${ }^{12,13}$. This finding suggests that the heart

55 rate-guided up-titration of VNS may be unnecessary and unrealistic in humans. To

56 prove this hypothesis, we need to examine whether VNS below the symptom threshold

57 yields any improvement in CHF. In this study, we investigated the impact of

58 subthreshold VNS that did not induce either symptoms or heart rate reduction on

59 cardiac function and CHF parameters in rats.

60

61 
Methods

62

63 Experiments and animal care were approved by the Committee on Ethics of Animal

64 Experiment, Kyushu University Graduate School of Medical Sciences. The

66 67 1985).

68

69

\section{Methods} investigation conforms to the Guide for the Care and Use of Laboratory Animals published by the US National Institutes of Health (NIH Publication No. 85-23, revised

\section{Animal preparation}

We anesthetized 8 week-old male Sprague-Dawley rats (SLC, Hamamatsu, Japan) with a mixture of isoflurane in oxygen-enriched air. We ligated the left anterior descending coronary artery to create large MI. One week after ligation, we performed echocardiography (SSA-380A, TOSHIBA, Japan) under anesthesia ${ }^{14}$. Rats with dilated left ventricle $(\mathrm{LV})(\mathrm{LV}$ diastolic dimension $\geq 9.5 \mathrm{~mm}$ ) and reduced $\mathrm{LV}$ ejection fraction (LVEF) were included in subsequent experiments (Table 1). We attached a pair of stainless steel electrodes to the right vagal nerve at the neck level and implanted a pulse generator (ANRE-100, Anpex, Japan) to stimulate the vagal nerve. We also implanted a telemetry system (TA11PA-C40, Data Sciences International, USA) and inserted the pressure sensing catheter in the abdominal aorta to record heart rate (HR) and blood pressure (BP).

\section{Protocol and titration of VNS}

One week after implantation, we randomized the rats into a VNS group $(n=41)$ and a sham group (Sham, $\mathrm{n}=16$ ). The VNS groups were divided into 3 groups depending on the voltage of VNS. 
Methods

We determined the symptom threshold by observing symptoms such as

87 respiratory twitching and abnormal behaviour while changing the voltage of VNS. We defined the maximum voltage as the voltage just below the threshold (Max; $n=14)$. We defined the half-maximum voltage as $1 / 2$ of Max (Half; $n=13$ ) and quarter-maximum voltage as $1 / 4$ of Max (Quarter; $n=14)$. Figure 1 shows the representative HR response of a rat during titration of VNS. VNS at Max, Half and Quarter did not reduce HR during VNS. The voltage just above Max evoked symptoms in all rats without HR reduction, while a higher voltage (Max +0.5 volts) significantly reduced HR. The

94 frequency was set at $20 \mathrm{~Hz}$, pulse width at $0.18 \mathrm{~ms}$, and duty cycle at $10 \mathrm{~s} / \mathrm{min}$. We titrated the VNS voltage once a week in every rat, readjusting the voltage if necessary.

96 Each rat received chronic VNS for 4 weeks.

\section{Echocardiography and hemodynamic studies}

At the end of the protocol (after 4 weeks of VNS), we anesthetized the rats with

100 isoflurane and recorded echocardiograms and hemodynamic under closed-chest

101 condition. After performing echocardiography, we inserted a $2 \mathrm{~F}$ catheter-tipped micromanometer (SPR-320: Millar Instruments, USA) into the LV via the right carotid artery and recorded blood pressure and LV pressure (LVP). We digitized LVP at $1 \mathrm{kHz}$ using a 16-bit analogue-to-digital converter (Power Lab 16/35: AD Instruments, NSW,

105 Australia) and stored in a dedicated laboratory computer system. We calculated the first

106 derivatives of LVP to estimate max $\mathrm{dp} / \mathrm{dt}$ and $\max -\mathrm{dp} / \mathrm{dt}$ as indexes of systolic function 107 and diastolic function, respectively. 
Methods

109 Neurohormonal studies

110 We sampled blood for measurements of hormone concentrations at the end of the

111 hemodynamic study. Plasma concentrations of norepinephrine and brain natriuretic

112 peptide (BNP) were measured.

113

114 Remodeling study and histological studies

115 After hemodynamic measurements, the heart and lung were excised, weighed, and then

116 dissected and fixed in $10 \%$ buffered formalin. The LV was sliced from the apex to base

117 into 4 transverse slices of $4-\mu \mathrm{m}$ thickness, and stained by Masson trichrome method. We

118 digitized histological images and estimated the size of MI using the ratio of the total

119 length of MI lesion relative to the total circumferential length in 4 slices $^{15}$.

120 We harvested the right vagal nerve from the site of electrode implantation. All

121 samples were fixed in $2.5 \%$ glutaraldehyde, cut into $4-\mu \mathrm{m}$ thick sections and stained

122 with toluidine blue. The number and diameter of myelinated fibers in the vagal trunk

123 were analysed under a microscope (Olympus, Japan). The fibers were classified as large

$124(\geq 3 \mu \mathrm{m})$ or small $(<3 \mu \mathrm{m})$.

125

126 Statistical Analysis

127 Results are expressed as mean \pm standard error of mean (SEM). One-way ANOVA was

128 used to compare each parameter in four experimental groups (Max, Half, Quarter, and

129 Sham). Post-hoc Dunnett test was then performed to identify which group differences

130 accounted for the significant overall ANOVA. A $p$ value less than 0.05 was considered

131 significant. 
Results

\section{Results}

\section{Alteration of VNS stimulation setting}

134 Shown in Table 2 are weekly alterations of electrode impedance, stimulation voltage,

135 and current. We derived the electrode impedance by applying a test voltage with a fixed

136 amplitude (5 volts) and measured the resultant current. Taking the ratio of the test

137 voltage to the current yielded the electrode impedance. The electrode impedance

138 increased gradually in all groups. In most cases, we had to increase the stimulation

139 voltage for titration. As a result, the stimulation current remained unchanged throughout

140 the observation period in all groups. Although we employed the same electrode and

141 surgical procedure to attach the electrode to the vagal nerve, minute variations such as

142 connective tissue growing between the vagal fibers and the electrode, uncontrollable

143 leakage of electricity and the involvement of sensory nerves in the vagal nerve and/or in

144 the electrode could not be avoided in some rats. In such cases, increasing in the

145 electrode impedance did not parallel the stimulation voltage of symptom threshold.

147 Effect of chronic VNS on 24-h averaged heart rate and blood pressure

148 Figure 2 shows the 24-h averaged HR and mean BP during 4 weeks of VNS. Half 149 reduced 24-h averaged HR significantly compared to Sham from 1 to 4 weeks of VNS, 150 while Max reduced HR at 3 and 4 weeks of VNS. The difference between Sham and

151 Half reached $40 \mathrm{bpm}$ at the end of the study $(p=0.003)$. Mean BP averaged over $24 \mathrm{~h}$

152 did not differ among 4 groups (Sham: $86.5 \pm 3.8$, Quarter: $84.1 \pm 1.6$, Half: $89.7 \pm 1.8$, 153 Max: $91.0 \pm 2.1 \mathrm{mmHg}$, not significant). 
Results

156

157

158

159

160

161

162

163

164

165

166

167

168

169

170

171

172

173

174

175

176

177

178

179

\section{Effect of VNS on LV function}

Figure 3 shows the effect of VNS on LV function. Half did not decrease LVDd (Half: $10.9 \pm 0.2$ vs. Sham: $11.1 \pm 0.1 \mathrm{~mm}$ ), while significantly decreased LVDs (Half: $9.1 \pm$ 0.2 vs. Sham: $9.8 \pm 0.2 \mathrm{~mm}, p<0.05)$. As a result, Half significantly preserved LVEF compared to Sham (Half: $37.9 \pm 3.1$ vs. Sham: $28.4 \pm 2.3 \%, p<0.05$ ). Furthermore, Half reduced LV end-diastolic pressure (LVEDP) by $6.7 \mathrm{mmHg}$ compared to Sham (Half: $17.5 \pm 2.0$ vs. Sham: $24.2 \pm 1.2 \mathrm{mmHg}, p<0.01$ ), and showed the highest maximum LV $+\mathrm{dP} / \mathrm{dt}$. (Sham: $5001 \pm 563$, Quarter: $4988 \pm 201$ Half: $5918 \pm 20$, Max: $5351 \pm 189 \mathrm{mmHg} / \mathrm{s}$; Half vs. Sham, $p<0.05)$ and the lowest minimum LV $-\mathrm{dP} / \mathrm{dt}$ (Sham: $-3632 \pm 188$, Quarter: $-3595 \pm 136$, Half: $-4342 \pm 309$, Max: $-3903 \pm 156$ $\mathrm{mmHg} / \mathrm{s}$; Half vs. Sham, $p<0.05)$.

\section{Effect of VNS on other parameters}

Table 3 shows the effect of VNS on infarct size, body weight, organ weights and neurohormonal factors. Infarct size and body weight were not different across groups at the completion of the VNS protocol. Max, Half and Quarter significantly reduced biventricular weight compared to Sham. Only Half significantly decreased lung weight compared to Sham, indicating improvement of pulmonary edema. Both Half and Max markedly decreased plasma BNP, a biomarker of aggravation of heart failure (Half: $157.4 \pm 25.7$ and Max: $177.8 \pm 27.6$ vs. Sham: $397.3 \pm 37.5 \mathrm{pg} / \mathrm{mL}$, both $p<0.01$ ). Half reduced plasma norepinephrine, a marker of sympathetic nerve activity, by more than 50\% compared to Sham (Half: $474.8 \pm 58.6$ vs. Sham: $936.8 \pm 126.2 \mathrm{pg} / \mathrm{mL}, p<0.01$ ). 
Results

\section{Histology of vagal nerve}

181 Figure 4 shows the effect of VNS on histological damage of vagal nerve. VNS did not

182 change the number of small fibers (Sham: $1222.7 \pm 293.9$, Quarter: 1086.8 \pm 151.2 ,

183 Half: $1224.4 \pm 108.6$, Max: $1312.5 \pm 98.3$ counts/bundle). However, Max significantly

184 decreased the number of large fibers (Max: $163.1 \pm 43.0$ vs. Sham: $360.0 \pm 61.6$

185 counts/bundle, $p<0.05)$. 
Discussion

\section{Discussion}

187 This is the first study to elucidate the dose response of VNS below the symptom 188 threshold, with respect to the effect on CHF. Major findings of this study are: (1)

189 up-titration of VNS evoked symptoms before the level that reduced heart rate; (2) A

190 VNS voltage at $50 \%$ of the symptom threshold (Half) significantly improved cardiac

191 remodelling and heart failure compared to other voltages; and (3) a voltage just below

192 the symptom threshold (Max) induced histological injury to vagal nerve fibers.

193

\section{Effect of VNS voltages below symptom threshold on heart failure}

195 We compared the effect of chronic VNS at three different voltages below the symptom

196 threshold. Max, Half and Quarter did not elicit symptoms such as pain and respiratory

197 twitching, and all three also did not reduce HR during stimulation, whereas the higher 198 voltage (Max +0.5 volts) evoked both HR reduction and symptoms. Although all three 199 voltages of VNS did not exhibit direct HR reduction during the period of titration, Half 200 had the lowest 24-h averaged HR among 4 groups all through 4 weeks of chronic VNS.

201 Since HR strongly correlates with the severity of heart failure ${ }^{16}$, this result indicates that

202 Half improved heart failure. As shown in Fig. 3 and Table 3, Quarter failed to improve any parameter of CHF, indicating that a certain intensity level of VNS is required to achieve beneficial effects. Half, which was the voltage at $50 \%$ of the symptom threshold,

205 clearly improved cardiac function, cardiac remodelling and heart failure, while Max

206 showed no significant improvement in these parameters except for biventricular weight

207 and plasma BNP. These data suggest that optimal stimulus dose is required to maximize 208 the beneficial effects of VNS for CHF. Using a stimulation intensity (current amplitude 209 of 0.1 to $0.13 \mathrm{~mA}$ ) sufficient to reduce heart rate, Li et al. ${ }^{3}$ showed that chronic VNS 


\section{Discussion}

210 improved cardiac function and survival in a rat model of heart failure after myocardial

211 infarction. In our study, we were not able to up-titrate VNS because of adverse effects.

212 At Max that did not decrease heart rate, we found no benefit of chronic VNS on

213 ventricular function. The difference in physiological response to VNS observed in Li's

214 and our study may be due to differences in electrode and stimulator ( $\mathrm{Li}$ et al. used a

215 constant current system). Thus, we cannot directly interpret and compare the two

216 findings.

217 Several possible mechanisms may explain why Half yielded better outcomes

218 than Max. As shown in Fig. 4, chronic electrical stimulation at the maximum voltage

219 caused significant neural fiber damage. Several modes of injury such as compressive ${ }^{17}$,

220 reperfusion $^{18,19}$ and electrical damage to peripheral nerves secondary to implantation

221 and electrical activation of electrodes have been reported ${ }^{20,21}$. In the present study,

222 physical damages caused by compression and electrode attachment to the vagal nerve

223 were conceivably the same in the four groups. We assume that the higher electrical

224 intensity of Max may have induced neural damage. As for histological investigation,

225 Agnew et al. ${ }^{20}$ showed that electrical stimulation of feline peroneal nerves reduced the

226 number of large myelinated fibers, whereas the smaller myelinated fibers were spared.

227 Their findings are consistent with our observation that the maximum subthreshold

228 voltage of VNS reduced the number of large fibers but not small fibers (Fig. 4). To

229 clarify if the histological neural damage parallels the function or sensitivity of vagal

230 nerve, we examined the HR response to VNS at the highest voltage (10 volts,

231 supramaximal) during titration as an indicator of loss of vagal nerve function. As shown

232 in Fig. 5, supramaximal VNS in Max significantly attenuated the HR responder

233 compared to other groups indicating that Max induced a functional deficit of vagal 


\section{Discussion}

nerve as well as an anatomical deficit. In addition, it is well known that the myelinated,

235 large vagal fibers play a pivotal role in cardio-protective effects of VNS. ${ }^{22}$ Thus,

236 electrical damage and loss of vagal myelinated fibers might have weakened the

237 beneficial effects of Max. Another possible factor is the sensitivity of vagal nerve. The

238 sensitivity and activity of vagal nerve vary daily or even hourly. Since we fixed the

239 voltage of Max just below the symptom threshold, we cannot rule out the possibility

240 that Max exceeded the threshold transiently. Persisting symptoms may evoke worsening

241 of respiratory rhythm and stress-induced sympathetic activation, and in turn lead to

242 aggravation of CHF.

244 The need for investigation of other parameters to optimize VNS

245 Apart from voltage, other stimulation parameters such as frequency, duration and duty

246 cycle should be considered to maximize the impact of VNS for CHF. In particular,

247 frequency should be another important factor. Saku et al. ${ }^{8}$ reported that VNS at $20 \mathrm{~Hz}$

248 induced marked respiratory inhibition via the vagal nerve afferent pathway. In addition,

249 we also evaluated how VNS frequency altered respiration, and clearly demonstrated that

250 VNS at $5 \mathrm{~Hz}$ delivered higher voltage without obvious respiratory inhibition compared

251 to VNS at $20 \mathrm{~Hz}^{23}$. These findings suggest that the frequency of VNS may alter the

252 symptom threshold. Further investigations are needed to decide the optimal combination

253 of stimulation parameters which balance the efficacy and adverse effects.

\section{Clinical implication}

256 Several clinical trials on VNS for CHF have been reported, and stimulation parameters

257 varied among trials. In clinical trials, stimulation-related symptoms limited up-titration 
Discussion

of VNS. De Ferrari et al. ${ }^{9}$ showed that adverse effects (discomfort and pain) prevented up-titration in $72 \%$ of the patients. In NECTAR-HF, the current amplitude used in VNS was less than half of the target current amplitude $(4 \mathrm{~mA})$ because of side effects, resulting in a low current amplitude of $1.42 \pm 0.80 \mathrm{~mA}^{10}$.

There have been no studies, including animal investigations, which examine the dose-efficacy relationship of VNS below the symptom threshold. We demonstrated that half of the maximum subthreshold voltage outperforms the maximum subthreshold voltage. In the open-labelled ANTHEM-HF study, the VNS current amplitude was set below the symptom threshold with a safety margin $(0.25 \mathrm{~mA})$ to insure that the therapy was well tolerated ${ }^{11}$. This dose setting may be a reason why VNS in patients with CHF achieved significant improvement of LV ejection fraction and exercise tolerance in ANTHEM-HF. Titration of VNS considering both intensity and safety would maximize the beneficial effects and minimize the adverse effects in CHF patients. Taken together, we cannot support the notion that the stronger intensity the better.

\section{Limitation}

There are several limitations in this study. First, in studying the dose effects of VNS, many stimulation parameters other than the voltage are involved, such as frequency, duty cycle, timing to the cardiac cycle and efferent/afferent nerve activation. In this study, we focused on the voltage. To develop VNS with maximum efficacy and minimum adverse effects, further investigations are needed to assess the effects of other stimulation parameters.

Second, since we focused on the impact of VNS on cardiac function and remodeling after myocardial infarction, we did not fully evaluate the mechanistic 


\section{Discussion}

insights of our findings. Zhang et al. ${ }^{13}$ reported that improvement of autonomic balance,

283 inhibition of the renin-angiotensin system and anti-inflammatory effects mediate the

284 therapeutic effects of VNS on CHF. It is conceivable that such mechanisms may also

285 contribute to the beneficial effects of low-dose VNS in this study. Further investigations

286 are needed to clarify the fundamental mechanism of low-dose VNS.

Third, we showed that the loss of vagal large fibers paralleled the beneficial effect of VNS on CHF at Max. Since the design of electrode and stimulus conditions in this study differ greatly from those in human, we should be very careful to extrapolate

290 this finding to VNS in patients. On the other hand, VNS evokes similar physiological

291 responses in rats and in humans. Therefore, the symptom guided up-titration method proposed in this study could serve as a practical indicator in determining the doses of

293 VNS in patients.

\section{Conclusion}

296 Optimal titration maximize the beneficial effects of VNS for CHF. A VNS voltage at $29750 \%$ of the symptom threshold significantly improved CHF. The proposed titration 298 procedure is potentially a practical clinical tool in determining the optimal dose of VNS. 
Acknowledgement

299

300

301

302

303 304 device.

\section{Acknowledgment}

The authors thank Ms. Meihua Li, Mr. Can Zheng and the staff of the Department of Cardiovascular Medicine of Kyushu University Graduate School of Medical Sciences for technical support. Additionally, the authors wish to thank Mr. Kazuo Shimizu and technical staffs of Anpex CO.,LTD. (Tokyo, Japan) for technical advice on the VNS

\section{Funding}

307 This work was supported by Grand-in-Aid from Japan Science and Technology Agency

308 (JST). Scientific Research (S) (23220013) from the Japan Society for the Promotion of

309 Science and Health and Labour Sciences Research Grant for Research on Medical

310 Devices for Improving Impaired QOL from the Ministry of Health Labour and Welfare

311 of Japan, Grant-in-Aid for Young Scientists (B) (15K19383) from the Japan Society for

312 the Promotion of Science and Grant-in-Aid for Young Scientists (B) (15K19386) from

313 the Japan Society for the Promotion of Science.

\section{Author Contributions}

316 Dr. Akiko Nishizaki contributed to conduct the whole study and wrote this manuscript.

317 Dr. Kazuo Sakamoto contributed to design of the work and revising the manuscript

318 critically for important intellectual content. Dr. Keita Saku contributed to interpretation

319 of data and revising the manuscript critically for important intellectual content. Dr.

320 Kazuya Hosokawa contributed to the design of the work and revising the manuscript

321 critically for important intellectual content. Dr. Takafumi Sakamoto contributed to 
Acknowledgement

322 interpretation of data and revising the manuscript critically for important intellectual

323 content. Dr. Yasuhiro Oga contributed to acquisition of data and drafting the manuscript.

324 Mr. Takuya Akashi contributed to acquisition of data and drafting the article. Mr.

325 Yoshinori Murayama contributed to analysis and interpretation of data and drafting the

326 article. Dr. Takuya Kishi contributed to analysis and interpretation of data and drafting

327 the manuscript. Dr. Tomomi Ide contributed to design of the work, interpretation of data,

328 and revising the manuscript critically for important intellectual content. Dr. Kenji

329 Sunagawa contributed to design of the work, interpretation of data and revising the

330 manuscript critically for important intellectual content. All authors approved this

331 manuscript to be published.

332 


\section{References}

1. Levy D, Kenchaiah S, Larson MG, et al. Long-term trends in the incidence of and survival with heart failure. N Engl J Med. 2002;347(18):1397-1402. doi:10.1056/NEJMoa020265.

2. Ponikowski P, Chua TP, Piepoli M, et al. Augmented Peripheral Chemosensitivity as a Potential Input to Baroreflex Impairment and Autonomic Imbalance in Chronic Heart Failure. Circulation. 1997;96(8):2586-2594. doi:10.1161/01.CIR.96.8.2586.

3. Li M, Zheng C, Sato T, Kawada T, Sugimachi M, Sunagawa K. Vagal Nerve Stimulation Markedly Improves Long-Term Survival after Chronic Heart Failure in Rats. Circulation. 2004;109(1):120-124. doi:10.1161/01.CIR.0000105721.71640.DA.

4. Olshansky B, Sabbah HN, Hauptman PJ, Colucci WS. Parasympathetic nervous system and heart failure pathophysiology and potential implications for therapy. Circulation. 2008;118(8):863-871. doi:10.1161/CIRCULATIONAHA.107.760405.

5. Tracey KJ. Physiology and immunology of the cholinergic antiinflammatory pathway. J Clin Invest. 2007;117(2):289-296. doi:10.1172/JCI30555.

6. Levy MN, Blattberg B. Effect of vagal stimulation on the overflow of norepinephrine into the coronary sinus during cardiac sympathetic nerve stimulation in the dog. Circ Res. 1976;38(2):81-84. doi:10.1161/01.RES.38.2.81.

7. Tsutsumi T, Ide T, Yamato M, et al. Modulation of the myocardial redox state by vagal nerve stimulation after experimental myocardial infarction. Cardiovasc Res. 2008;77(4):713-721. doi:10.1093/cvr/cvm092.

8. Saku K, Kishi T, Sakamoto K, et al. Afferent vagal nerve stimulation resets baroreflex neural arc and inhibits sympathetic nerve activity. Physiol Rep. 2014;2(9):e12136-e12136. doi:10.14814/phy2.12136. 
References

9. De Ferrari GM, Crijns HJGM, Borggrefe M, et al. Chronic vagus nerve stimulation: A new and promising therapeutic approach for chronic heart failure. Eur Heart J. 2011;32(7):847-855. doi:10.1093/eurheartj/ehq391.

10. Zannad F, De Ferrari GM, Tuinenburg a. E, et al. Chronic vagal stimulation for the treatment of low ejection fraction heart failure: results of the NEural Cardiac TherApy foR Heart Failure (NECTAR-HF) randomized controlled trial. Eur Heart J. 2015;36(7):425-433. doi:10.1093/eurheartj/ehu345.

11. Premchand RK, Sharma K, Mittal S, et al. Autonomic Regulation Therapy via Left or Right Cervical Vagus Nerve Stimulation in Patients With Chronic Heart Failure: Results of the ANTHEM-HF Trial. J Card Fail. 2014;20(11):808-816. doi:10.1016/j.cardfail.2014.08.009.

12. Sabbah HN, Ilsar I, Zaretsky A, Rastogi S, Wang M, Gupta RC. Vagus nerve stimulation in experimental heart failure. Heart Fail Rev. 2011;16(2):171-178. doi:10.1007/s10741-010-9209-z.

13. Zhang Y, Popović ZB, Bibevski S, et al. Chronic vagus nerve stimulation improves autonomic control and attenuates systemic inflammation and heart failure progression in a canine high-rate pacing model. Circ Hear Fail. 2009;2(6):692-699. doi:10.1161/CIRCHEARTFAILURE.109.873968.

14. Litwin SE, Katz SE, Morgan JP DP. Serial echocardiographic assessment of left ventricular geometry and function after large myocardial infarction in the rat. Circulation. 1994;89(1):345-354. doi:10.1097/00004872-200502000-00032.

15. Pfeffer MA, Pfeffer JM, Steinberg C, Finn P. Survival after an experimental myocardial infarction: beneficial effects of long-term therapy with captopril. Circulation. 1985;72(2):406-412. doi:10.1161/01.CIR.72.2.406.

16. Dobre D, Borer JS, Fox K, et al. Heart rate: A prognostic factor and therapeutic target in chronic heart failure. The distinct roles of drugs with heart rate-lowering properties. Eur J Heart Fail. 2014;16(1):76-85. doi:10.1093/eurjhf/hft129.

17. Dyck PJ, Lais a C, Giannini C, Engelstad JK. Structural alterations of nerve during cuff compression. Proc Natl Acad Sci U S A. 1990;87(24):9828-9832. doi:10.1073/pnas.87.24.9828. 
References

18. Wang Y, Kawamura N, Schmelzer JD, Schmeichel AM, Low PA. Decreased peripheral nerve damage after ischemia-reperfusion injury in mice lacking TNF-alpha. J Neurol Sci. 2008;267(1-2):107-111. doi:10.1016/j.jns.2007.10.004.

19. Iida H, Nagasaka T, Shindo K, Shiozawa Z. Effect of the free radical scavenger edaravone on peripheral nerve ischemia-reperfusion injury. Muscle Nerve. 2009;40(4):582-588. doi:10.1002/mus.21388.

20. Agnew WF, McCreery DB, Yuen TG, Bullara L a. Histologic and physiologic evaluation of electrically stimulated peripheral nerve: considerations for the selection of parameters. Ann Biomed Eng. 1989;17(1):39-60. doi:10.1007/BF02364272.

21. Cohen ML, Georgievskaya Z. Histopathology of the stimulated Vagus nerve: Primum non nocere. Heart Fail Rev. 2011;16(2):163-169. doi:10.1007/s10741-010-9182-6.

22. Nosaka S, Yasunaga K, Kawano M. Vagus cardioinhibitory fibers in rats. Pflugers Arch. 1979;379(3):281-285. doi:10.1007/BF00581432.

23. Saku K, Nishizaki A, Kishi T, Ide T, Sunagawa K. Stimulus frequency differences in respiratory inhibition is negligible to optimize vagal nerve stimulation (VNS) for heart failure. JSMBE Proceeding. 2014;52:84-85. doi:10.11239/jsmbe.52.O-84. 
Figure Legends

\section{Figure Legends}

Figure 1. Direct effect of vagal nerve stimulation (VNS) on heart rate (HR).

Max, voltage just below the symptom threshold; Half, half of Max; Quarter, quarter of Max; Sham, sham-stimulation. Subthreshold VNS up to Max did not reduce HR during stimulation. A higher symptom-evoking voltage (Max +0.5 volts) decreased HR.

Figure 2. Effects of vagal nerve stimulation (VNS) on 24-h averaged heart rate (HR) and 24-h mean blood pressure (BP) during 4 weeks of chronic VNS.

Max, voltage just below the symptom threshold; Half, half of Max; Quarter, quarter of Max; Sham, sham-stimulation. (A) Half significantly decreased HR compared to Sham during 4 weeks of VNS. (B) Mean BP did not differ among 4 groups. Data are expressed as mean \pm SEM. ${ }^{*} p<0.05,{ }^{*} p<0.01$ vs. Sham at the same period; $\dagger p<$ 0.05 vs. Sham at 0 week; n.s., not significant

Figure 3. Effects of vagal nerve stimulation (VNS) on left ventricular (LV) function.

Max, voltage just below the symptom threshold; Half, half of Max; Quarter, quarter of Max; Sham, sham-stimulation; LVDd, left ventricular end-diastolic dimension; LVDs, left ventricular end-systolic dimension; LVEF, left ventricular ejection fraction; LVEDP, 
Figure Legends

LV end-diastolic pressure; LV, left ventricle. Half significantly increased LVEF. Half decreased LVEDP, and showed the highest maximum $+\mathrm{LV} \mathrm{dP/dt}$ and the lowest minimum LV -dP/dt. Data are expressed as mean \pm SEM. ${ }^{*} p<0.05,{ }^{* *} p<0.01$ vs. Sham.

Figure 4. Effect of vagal nerve stimulation (VNS) on histology of vagal nerve.

Max, voltage just below the symptom threshold; Half, half of Max; Quarter, quarter of Max; Sham, sham-stimulation. (A) VNS at all three voltages did not decrease the number of small fibers. (B) Max decreased the number of large fibers of vagal nerve. Data are expressed as mean \pm SEM. ${ }^{*} p<0.05$ vs. Sham.

Figure 5. The impact of acute supramaximal VNS (10-volts) on heart rate (HR) among four groups.

Max, voltage just below the symptom threshold; Half, half of Max; Quarter, quarter of Max; Sham, sham-stimulation. We defined the HR responder as a rat showing the HR reduction of over $10 \mathrm{bpm}$ from the baseline, and counted the number of responders once a week in each group. We estimated the rate of HR responder among four groups by the Kaplan-Meier method and compared by log-rank test. Max halved the responder rate 
Figure Legends

after 4 weeks. On the contrary, the responder rate remained unchanged in Quarter or Half (Sham; 100, Quarter; 100, Half; 93.3 and Max; 52.6\% in 4-week after VNS, $p=0.01)$. 
$\underline{\mathrm{HR}}$ (bpm)

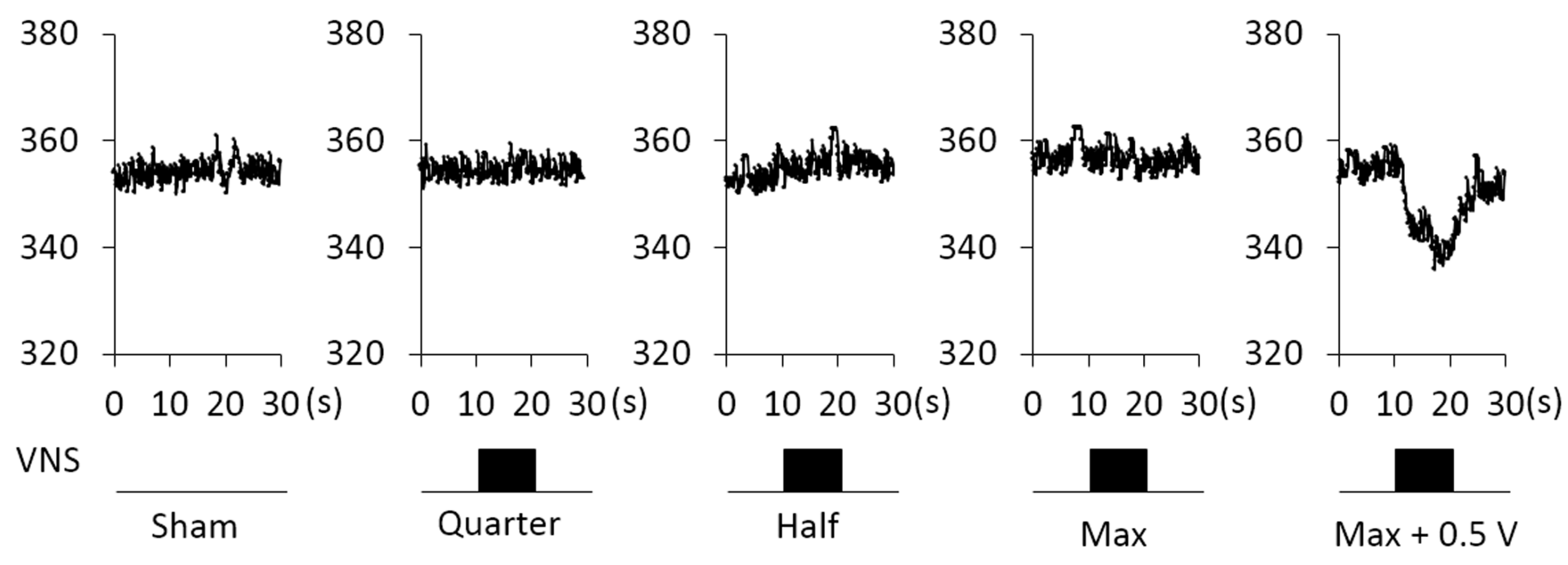


A 24-hour heart rate (bpm)

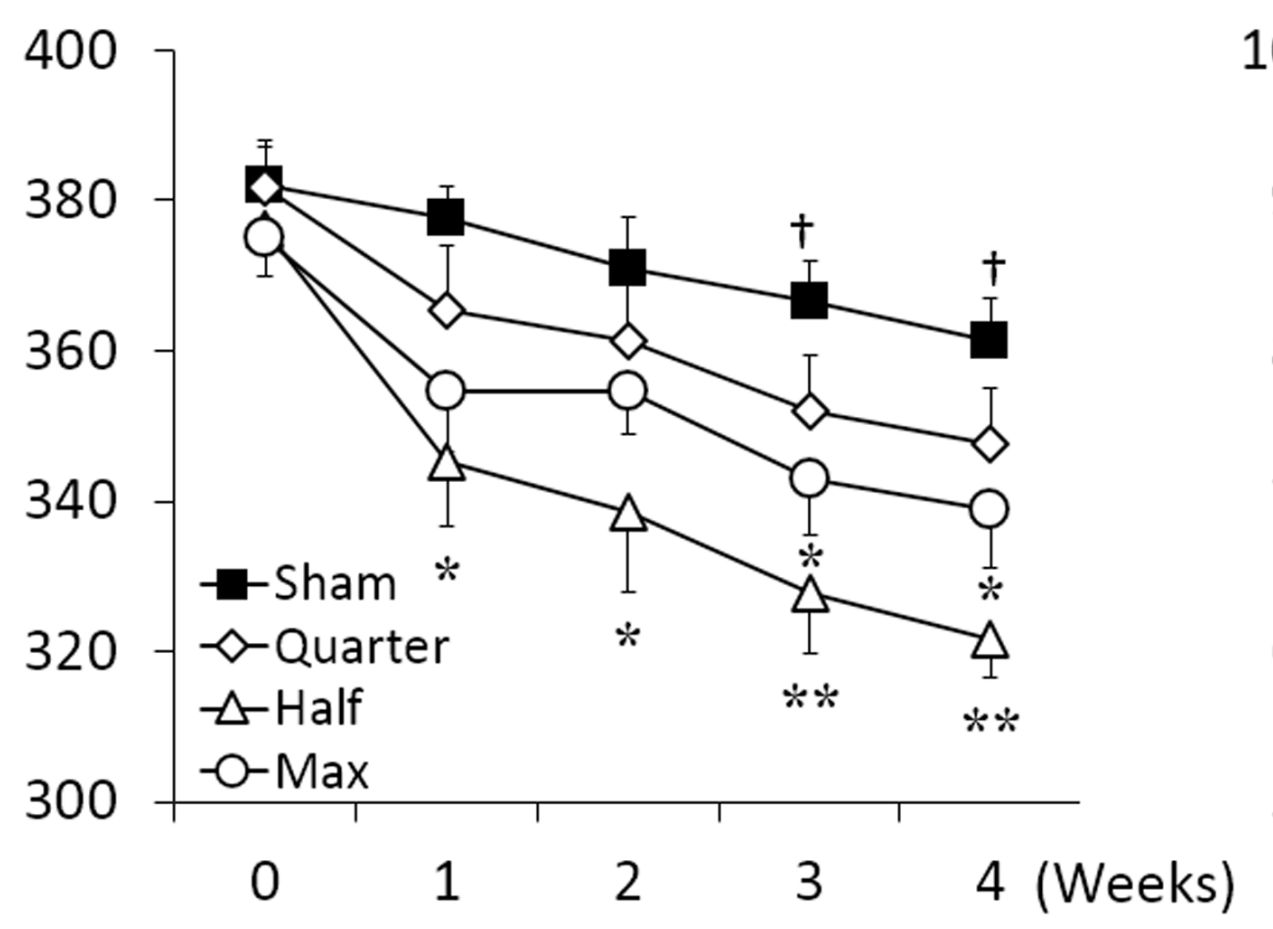

B 24-hour mean BP (mmHg)

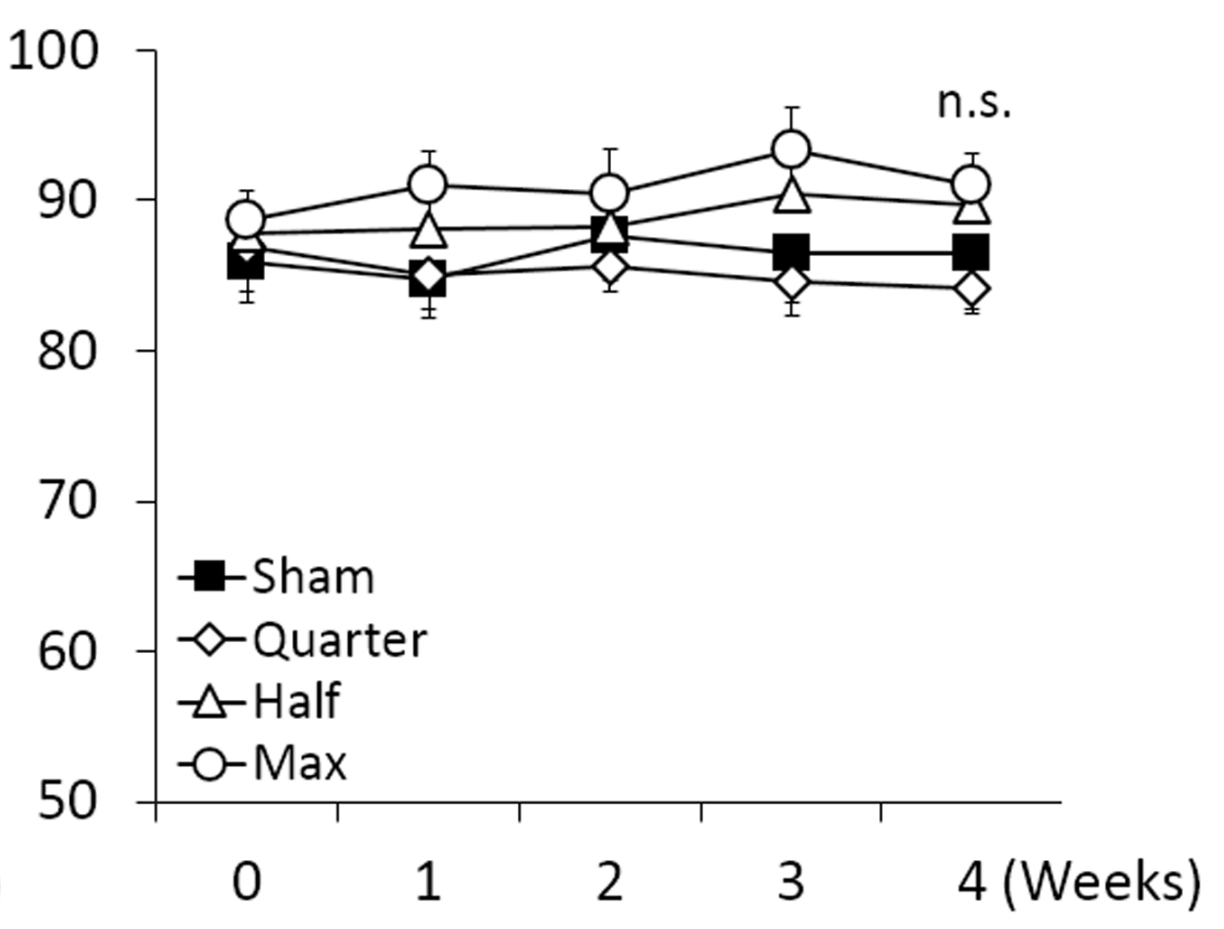



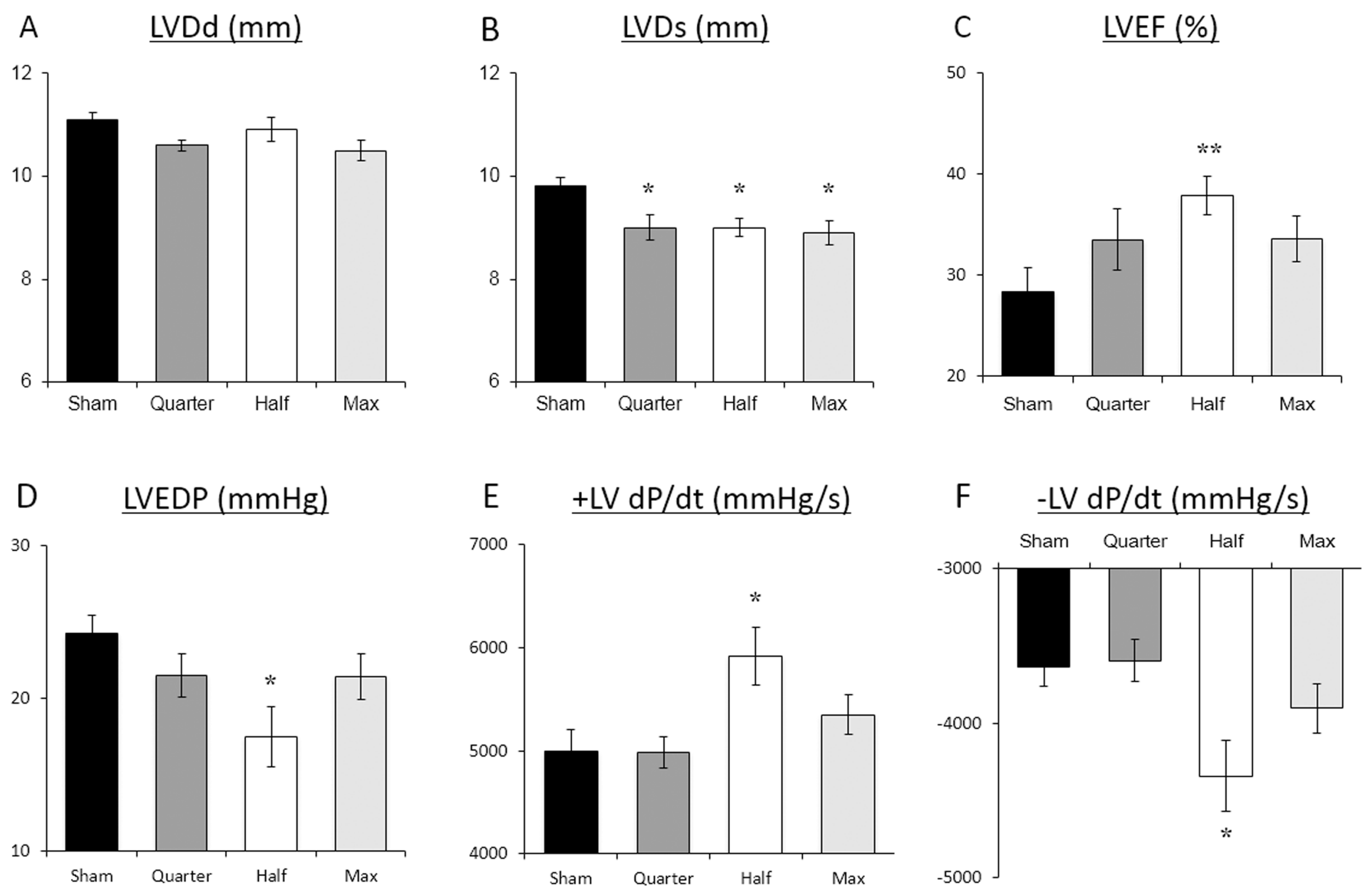
A Small fibers (counts/bundle)

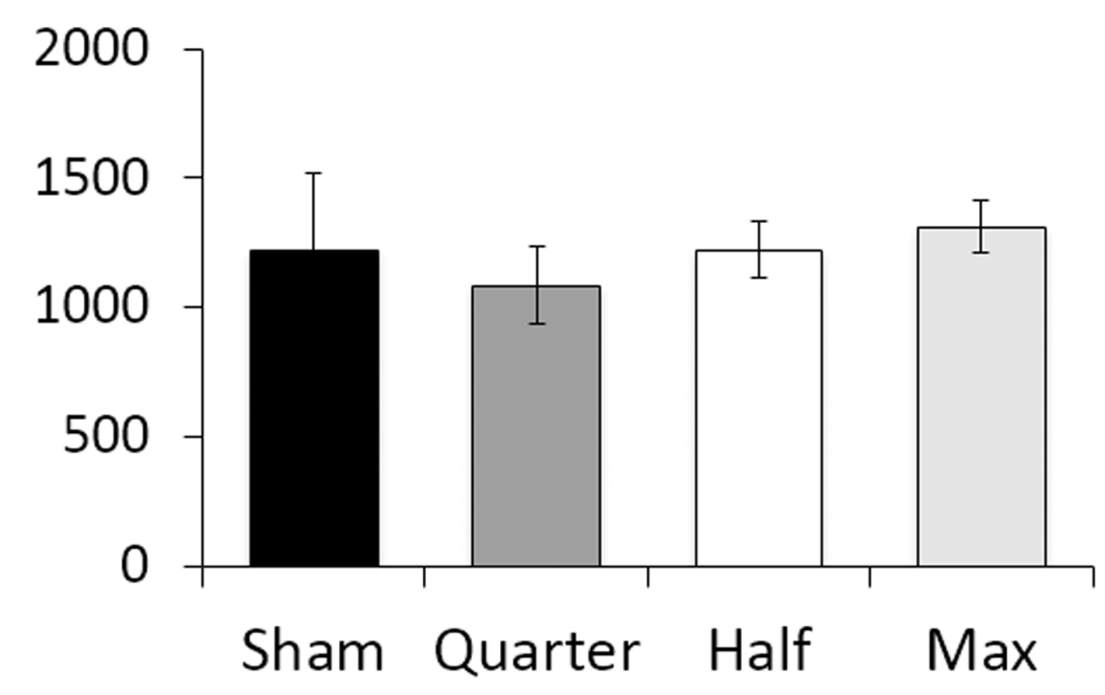

B Large fibers (counts/bundle)

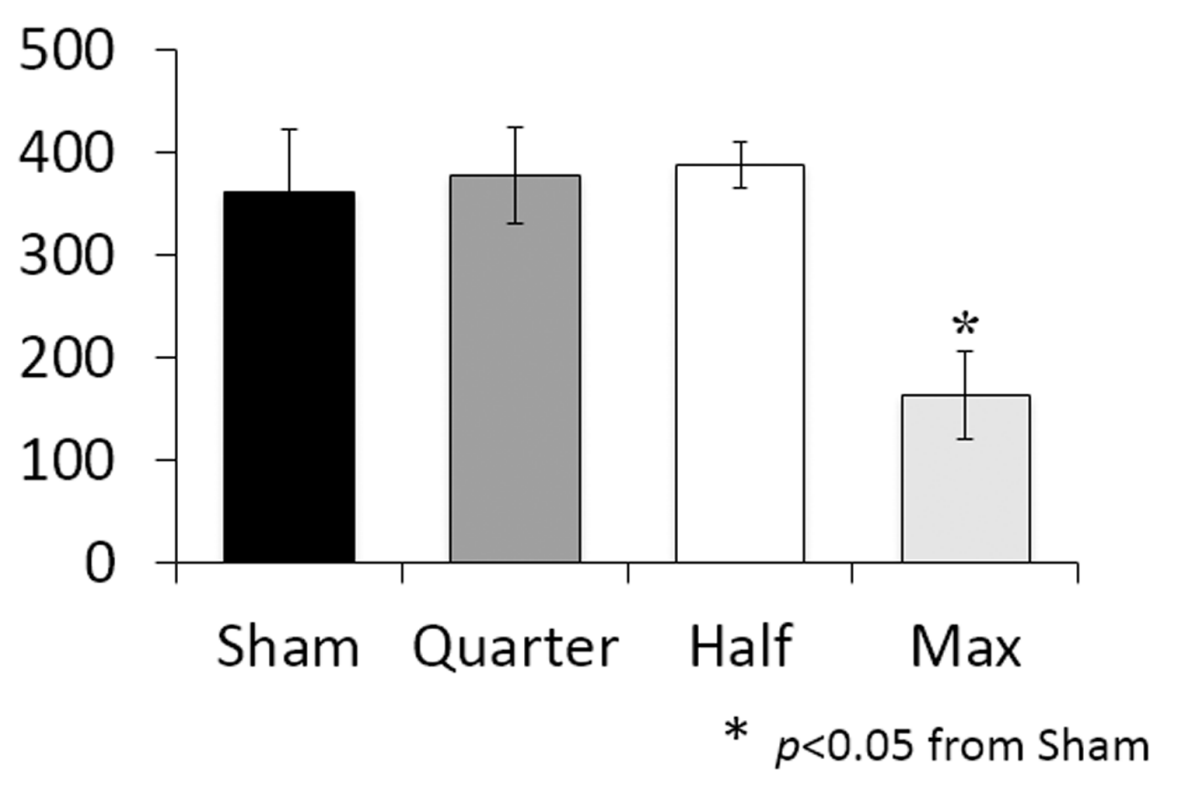




\section{$\underline{H R}$ responders to supramaximal VNS}

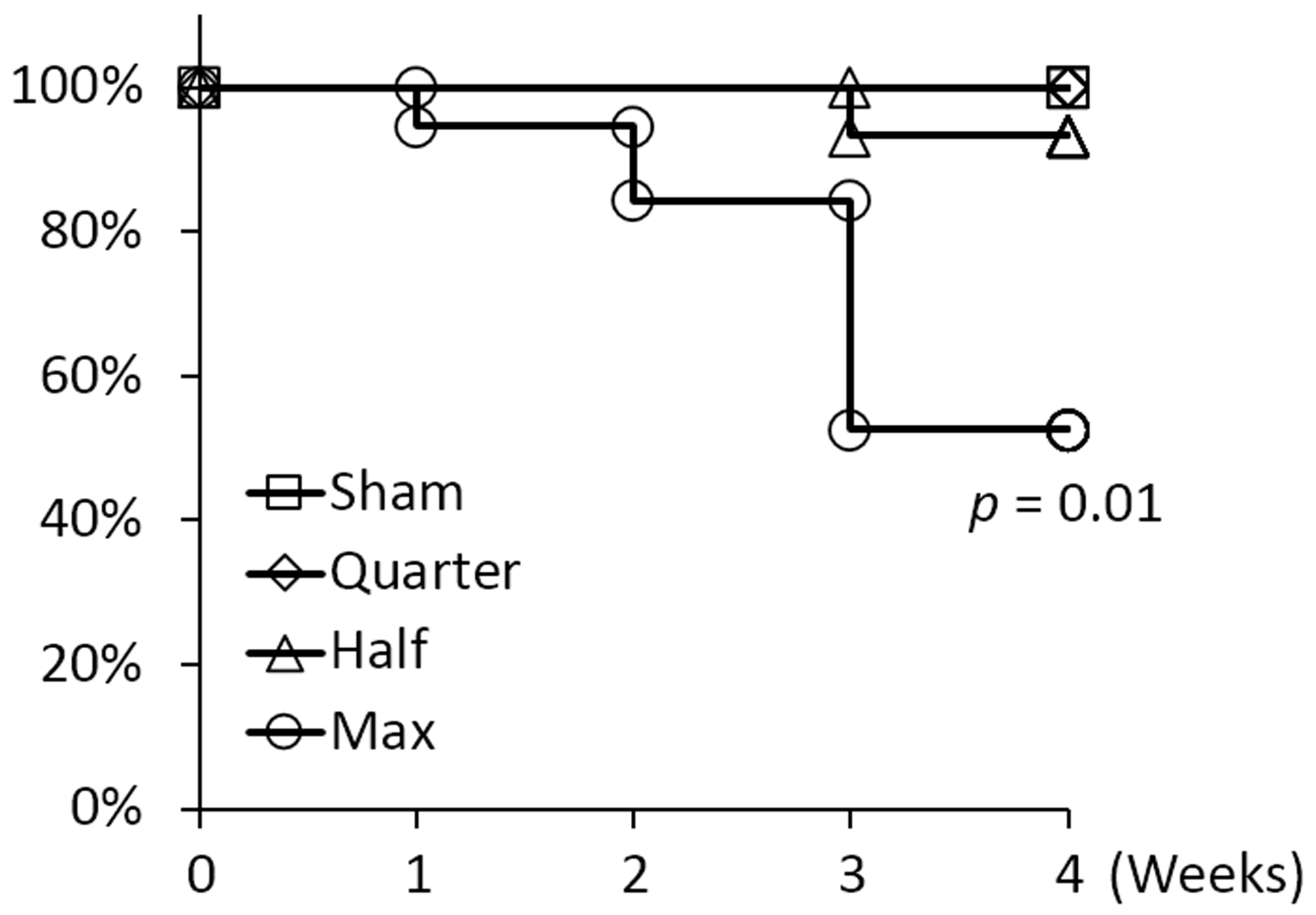


Table 1. Body weight and echocardiographic measurements at baseline

\begin{tabular}{c|cccc}
\hline & Sham & Quarter & Half & Max \\
\hline BW, g & $275.5 \pm 4.8$ & $264.4 \pm 7.7$ & $278.2 \pm 4.7$ & $268.8 \pm 8.4$ \\
LVDd, mm & $9.7 \pm 0.1$ & $9.6 \pm 0.1$ & $9.8 \pm 0.1$ & $9.6 \pm 0.1$ \\
LVDs, mm & $7.9 \pm 0.2$ & $7.9 \pm 0.1$ & $8.0 \pm 0.2$ & $7.9 \pm 0.1$ \\
LVEF, \% & $41.8 \pm 1.9$ & $41.7 \pm 1.8$ & $42.0 \pm 1.4$ & $41.6 \pm 1.8$ \\
\hline
\end{tabular}

Legend:

In each parameter (BW, LVDd, LVDs and LVEF), there were no significant difference among four groups. Max, voltage just below the symptom threshold; Half, half of Max; Quarter, quarter of Max; Sham, sham-stimulation; BW, body weight (g). LVDd, left ventricular end-diastolic dimension (mm). LVDs, left ventricular end-systolic dimension (mm). LVEF, left ventricular ejection fraction (\%). Data are expressed as mean \pm SEM 
Table 2. Weekly changes in VNS setting

\begin{tabular}{|c|c|c|c|c|c|c|c|c|c|c|c|c|c|c|c|}
\hline \multirow[b]{2}{*}{ weeks } & \multicolumn{5}{|c|}{ Impedance $(\mathrm{k} \Omega)$} & \multicolumn{5}{|c|}{ Stimulation Voltage (volts) } & \multicolumn{5}{|c|}{ Stimulation Current (mA) } \\
\hline & 0 & 1 & 2 & 3 & 4 & 0 & 1 & 2 & 3 & 4 & 0 & 1 & 2 & 3 & 4 \\
\hline Quarter & $13.2 \pm 1.0$ & $25.0 \pm 7.0$ & $31.8 \pm 10.1$ & $29.3 \pm 7.4$ & $30.2 \pm 7.3$ & $0.5 \pm 0.1$ & $0.5 \pm 0.1$ & $0.7 \pm 0.1$ & $0.8 \pm 0.1$ & $0.8 \pm 0.1$ & $0.03 \pm 0.007$ & $70.03 \pm 0.003$ & $0.03 \pm 0.003$ & $0.03 \pm 0.004$ & $0.03 \pm 0.003$ \\
\hline Half & $14.5 \pm 1.0$ & $20.9 \pm 1.7$ & $23.1 \pm 2.0$ & $25.9 \pm 4.3$ & $32.0 \pm 6.9$ & $1.1 \pm 0.2$ & $1.4 \pm 0.3$ & $1.9 \pm 0.5$ & $1.9 \pm 0.4$ & $2.0 \pm 0.4$ & $0.08 \pm 0.01$ & $0.08 \pm 0.02$ & $0.08 \pm 0.02$ & $0.07 \pm 0.01$ & $0.08 \pm 0.02$ \\
\hline $\operatorname{Max}$ & $17.9 \pm 1.9$ & $24.3 \pm 2.2$ & $26.6 \pm 3.2$ & $33.8 \pm 4.7$ & $39.6 \pm 9.2$ & $1.8 \pm 0.3$ & $2.3 \pm 0.5$ & $2.9 \pm 0.4$ & $3.7 \pm 0.7$ & $3.7 \pm 0.7$ & $0.11 \pm 0.03$ & $30.11 \pm 0.03$ & $0.11 \pm 0.01$ & $0.10 \pm 0.01$ & $0.10 \pm 0.01$ \\
\hline
\end{tabular}

Legend:

Weekly changes in electrode impedance, stimulation voltage, and current at each titration period. We derived the electrode impedance every week (see text for details). After knowing the electrode impedance, we stimulated the vagal nerve at various voltage amplitudes.

The ratio of stimulation voltage to the electrode impedance yielded the stimulation current. Max, voltage just below the symptom

threshold; Half, half of Max; Quarter, quarter of Max; Sham, sham-stimulation. Data are expressed as mean \pm SEM. 
Table 3. Effects of vagal nerve stimulation (VNS) on other parameters.

\begin{tabular}{c|cccc}
\hline & Sham & Quarter & Half & Max \\
\hline MI size, \% & $46.9 \pm 1.2$ & $51.8 \pm 2.3$ & $48.0 \pm 1.9$ & $48.8 \pm 1.6$ \\
BW, g & $396.4 \pm 9.7$ & $382.6 \pm 10.8$ & $405.5 \pm 13.2$ & $382.6 \pm 8.5$ \\
Biventricular & $3.7 \pm 0.11$ & $3.4 \pm 0.06^{*}$ & $3.2 \pm 0.11^{* *}$ & $3.2 \pm 0.12^{* *}$ \\
weight, g/kg & $9.7 \pm 0.3$ & $9.4 \pm 0.8$ & $6.7 \pm 0.8^{* *}$ & $8.3 \pm 0.5$ \\
Lung weight, g/kg & $397.3 \pm 37.5$ & $372.9 \pm 48.2$ & $157.4 \pm 25.7^{* *}$ & $177.8 \pm 27.6^{* *}$ \\
BNP, pg/mL & & & & $631.9 \pm 74.4$ \\
NE, $\mathrm{pg} / \mathrm{mL}$ & $936.8 \pm 126.2$ & $932.6 \pm 179.9$ & $474.8 \pm 58.6^{* *}$ & $631.9 \pm$ \\
\hline
\end{tabular}

Legend:

The effect of vagal nerve stimulation (VNS) on infarct ratio (MI size, normalized by LV circumferential length), body weight (BW), biventricular weight (normalized by body weight), lung weight (normalized by body weight), plasma brain natriuretic peptide (BNP) and plasma norepinephrine (NE). Infarct size and body weight were not different across groups at the completion of the VNS protocol. Max, voltage just below the symptom threshold; Half, half of Max; Quarter, quarter of Max; Sham, sham-stimulation. Data are expressed as mean \pm SEM. $* p<0.05, * * p<0.01$ vs. Sham. 